\title{
On Dimensionsgrad, resolutions, and chainable continua
}

by

\author{
Michael G. Charalambous (Karlovassi) and \\ Jerzy Krzempek (Gliwice)
}

\begin{abstract}
There exist very few examples of spaces exhibiting pathologies of Brouwer's Dimensionsgrad, Dg.

For each natural number $n \geq 1$ and each pair of ordinals $\alpha, \beta$ with $n \leq \alpha \leq \beta \leq \omega\left(\mathfrak{c}^{+}\right)$, where $\omega\left(\mathfrak{c}^{+}\right)$is the first ordinal of cardinality $\mathfrak{c}^{+}$, we construct a continuum $S_{n, \alpha, \beta}$ such that

(a) $\operatorname{dim} S_{n, \alpha, \beta}=n$;

(b) $\operatorname{trDg} S_{n, \alpha, \beta}=\operatorname{trDg} S_{n, \alpha, \beta}=\alpha$;

(c) trind $S_{n, \alpha, \beta}=\operatorname{trInd}_{0} S_{n, \alpha, \beta}=\beta$;

(d) if $\beta<\omega\left(\mathbf{c}^{+}\right)$, then $S_{n, \alpha, \beta}$ is separable and first countable;

(e) if $n=1$, then $S_{n, \alpha, \beta}$ can be made chainable or hereditarily decomposable;

(f) if $\alpha=\beta<\omega\left(\mathfrak{c}^{+}\right)$, then $S_{n, \alpha, \beta}$ can be made hereditarily indecomposable;

(g) if $n=1$ and $\alpha=\beta<\omega\left(\mathfrak{c}^{+}\right)$, then $S_{n, \alpha, \beta}$ can be made chainable and hereditarily indecomposable.
\end{abstract}

In particular, we answer the question raised by Chatyrko and Fedorchuk whether every non-degenerate chainable space has Dimensionsgrad equal to 1. Moreover, we establish results that enable us to compute the Dimensionsgrad of a number of spaces constructed by Charalambous, Chatyrko, and Fedorchuk.

1. Introduction. In this paper all spaces are completely regular and $T_{1}$, and a continuum is a non-empty, compact connected space. Let $A, B$ be disjoint closed subsets of a space $X$ and $C$ a closed subset of $X$ disjoint from $A \cup B$. Then $C$ is called a (zero) partition between $A$ and $B$ if $(C$ is a zero subset of $X$ and) there are disjoint open subsets $U, V$ of $X$ such that $A \subset U, B \subset V$ and $X \backslash C=U \cup V$; and $C$ is called a (zero) cut between $A$ and $B$ if ( $C$ is a zero subset of $X$ and) every continuum that meets both $A$ and $B$ meets $C$. Evidently, every (zero) partition is a (zero) cut.

The definitions of the dimension functions Ind, $\mathrm{Ind}_{0}, \mathrm{Dg}$, and $\mathrm{Dg}_{0}$ are quite similar: Ind $X, \operatorname{Ind}_{0} X, \operatorname{Dg} X$, or $\operatorname{Dg}_{0} X$ equals -1 iff $X=\emptyset$. For a

2010 Mathematics Subject Classification: Primary 54F45; Secondary 54F15.

Key words and phrases: Dimensionsgrad, inductive dimension, non-coinciding dimensions, chainable continuum, resolution, atomic map, fully closed map. 
non-empty normal space $X$, Ind $X$ (respectively, $\operatorname{Ind}_{0} X, \operatorname{Dg} X, \operatorname{Dg}_{0} X$ ) is the smallest non-negative integer $n$ for which between any pair of disjoint closed sets $A$ and $B$ of $X$, there is a partition (respectively, zero partition, cut, zero cut) $C$ with Ind $C$ (respectively, $\left.\operatorname{Ind}_{0} C, \operatorname{Dg} C, \operatorname{Dg}_{0} C\right) \leq n-1$, if such an integer exists. If no such integer exists, we set Ind $X$ (respectively, $\left.\operatorname{Ind}_{0} X, \operatorname{Dg} X, \operatorname{Dg}_{0} X\right)=\infty$. If in the above definition of Ind, we stipulate that the set $A$ is a singleton, we obtain the definition of ind. In [5, 6, 22 we used Ind 0 as a tool for estimating ind and Ind. Dg or Dimensionsgrad is Brouwer's original definition of dimension [3]. $\mathrm{Dg}_{0}$, which is introduced here, is a useful tool for computing Dimensionsgrad.

Transfinite extensions of the above dimension functions are obtained in the usual manner. Thus, for example, if $X$ is a non-empty normal space, $\operatorname{trDg}_{0} X$ is the smallest ordinal $\alpha$ for which between any pair of disjoint closed sets $A$ and $B$ of $X$, there is a zero cut $C$ with $\operatorname{trDg}_{0} C<\alpha$, if such an ordinal exists. If no such ordinal exists, we set $\operatorname{trDg}_{0} X=\infty$. Evidently, trind $\leq$ trInd $\leq \operatorname{trInd}_{0}, \mathrm{Dg} \leq$ Ind and $\operatorname{trDg} \leq \operatorname{trDg}_{0} \leq$ trInd $_{0}$. Hence, if trind $X=\operatorname{trInd}_{0} X$, then trind $X=\operatorname{trInd} X=\operatorname{trInd}_{0} X$.

It was thought for a surprisingly long time that Dg agrees with Ind on locally connected separable metric spaces. However, for any given $n>1$, Fedorchuk and van Mill [20] constructed an $n$-dimensional locally connected Polish space with Dg equal to 1 . On the positive side, we have the equality dim $=\mathrm{Dg}$ for compact metric spaces by Fedorchuk, Levin, and Shchepin [19], and the inequality $\operatorname{dim} \leq \mathrm{Dg}$ for compact Hausdorff spaces by Fedorchuk [16]. In the same paper, Fedorchuk presented a first countable and separable compact space with $\operatorname{dim}=2$ and $\mathrm{Dg}=\infty$. In another paper [17], he presented an example of a first countable and separable compact space $B$ with $2=\operatorname{dim} B<\operatorname{Dg} B=3<\operatorname{ind} B=\operatorname{Ind} B=4$. Our aim in this paper is to obtain a clearer picture of the gap between $\operatorname{dim}$ and $\mathrm{Dg}$, as well as the gap between Dg and Ind, on the class of compact spaces.

In [11] Chatyrko and Fedorchuk showed that the chainable spaces $I_{2}, I_{3}$ of Chatyrko [8], which satisfy ind $I_{2}=\operatorname{Ind} I_{2}=2$ and ind $I_{3}=\operatorname{Ind} I_{3}=3$, have Dimensionsgrad equal to 1 . They then raised the question whether $\operatorname{Dg} X=1$ for every non-degenerate chainable continuum $X$. In the same paper, they constructed a compact space with $\operatorname{dim}=1<\operatorname{Dg}=2<$ ind $=$ Ind $=3$.

In [5] Charalambous presented a chainable space $X_{n}, n=1,2, \ldots$, with $1 \leq \operatorname{Dg} X_{n}<\operatorname{ind} X_{n}=\operatorname{Ind} X_{n}=n$, leaving the precise value of $\operatorname{Dg} X_{n}$ an open problem for $n>2$. We show that $\operatorname{Dg} X_{n}=1$.

In 1968 Fedorchuk [15] invented the method of resolutions, and constructed the first examples of first-countable and separable compact spaces with non-coinciding dimensions $\operatorname{dim}$ and ind. For the $n$-dimensional sphere $S^{n}$ and the $n$-dimensional torus $T^{n}$, he constructed first-countable separable compact spaces $R_{T^{n}}\left(S^{n}\right)$, proved that $\operatorname{dim} R_{T^{2}}\left(S^{2}\right)=2$ and $3 \leq$ 
ind $R_{T^{2}}\left(S^{2}\right) \leq 4$, and stated that $\operatorname{dim} R_{T^{n}}\left(S^{n}\right)=n$ and $2 n-1 \leq \operatorname{ind} R_{T^{n}}\left(S^{n}\right)$ $\leq 2 n$. The space $B$ with $\mathrm{Dg}=3$ mentioned above is a ring-like resolution of the form $R_{T^{2}}\left(S^{2}\right)$. We prove that, in fact, $\operatorname{Dg} R_{T^{n}}\left(S^{n}\right)=2 n-1$ whenever $R_{T^{n}}\left(S^{n}\right)$ is ring-like.

2. Preliminaries: continua, maps, and dimensions. In this paper a map means a continuous function. The interval $[0,1]$ is denoted by $\mathbb{I}$, its cardinality by $\mathfrak{c}$, the real numbers by $\mathbb{R}$, and the set of natural numbers by $\mathbb{N}$. For a cardinal number $\mathfrak{m}, \omega(\mathfrak{m})$ denotes the least ordinal of cardinality $\mathfrak{m}$. For terms not explicitly defined here, the reader is directed to the monographs by Engelking [13, 14] and van Mill [26].

A continuum is said to be decomposable if it is the union of two proper subcontinua; otherwise, it is called indecomposable. A subcontinuum $P$ of a space $X$ is said to be terminal if every continuum $Q \subset X$ that meets $P$ satisfies $P \subset Q$ or $Q \subset P$. A space $X$ is hereditarily indecomposable, abbreviated to HI, if none of its subcontinua is decomposable, or equivalently, if each of its subcontinua is terminal. $X$ is hereditarily decomposable, abbreviated to $\mathrm{HD}$, if each of its non-degenerate subcontinua is decomposable.

If $f: X \rightarrow Y$ is a function and $A \subset X$, the small image of $A$ is defined as

$$
f^{\#}(A)=\left\{y \in Y: f^{-1}(y) \subset A\right\}=Y \backslash f(X \backslash A) .
$$

A map $f: X \rightarrow Y$ is said to be

- monotone if every point-inverse under $f$ is a continuum;

- atomic if every point-inverse under $f$ is a terminal subcontinuum of $X$;

- ring-like if for each point $x \in X$ and each open set $U \ni x$, every neighborhood $V$ of $f(x)$ contains an open set $V^{\prime} \ni f(x)$ such that bd $V^{\prime} \subset f^{\#}(U)$;

- fully closed if for each point $y \in Y$ and each finite covering of $f^{-1}(y)$ by sets $U_{1}, \ldots, U_{n}$ open in $X$, the set $\{y\} \cup \bigcup_{i=1}^{n} f^{\#}\left(U_{i}\right)$ is a neighborhood of $y$.

Note that monotone maps and atomic maps are surjective since any continuum is, by definition, non-empty. As is easily checked, a closed monotone map $f: X \rightarrow Y$ is atomic iff $P=f^{-1} f(P)$ for each continuum $P \subset X$ such that $f(P)$ is non-degenerate.

2.1. Proposition. Let $f: X \rightarrow Y$ be a closed monotone map, and $A, B, F \subset X$ be closed sets with pairwise disjoint images under $f$. If $F$ is a cut in $X$ between $A$ and $B$, then $f(F)$ is a cut in $Y$ between $f(A)$ and $f(B)$. The converse is true if $f$ is moreover atomic.

2.2. Remarks. (1) Suppose that $f: X \rightarrow Y$ is a closed ring-like map, and $P \subset X$ is closed. It is easily shown that if $f(P)$ is connected and contains more than one point, then $P=f^{-1} f(P)$ (see [18, II.1.15]). We shall 
frequently use this fact in the following form: If $Q \subset f(P)$ is connected, and contains more than one point, then $f^{-1}(Q) \subset P$. It follows that if $Y=f(X)$ is connected and contains more than one point, then $f$ is irreducible.

(2) If $f: X \rightarrow Y$ is a fully closed map, then $f^{\#}(U)$ is open in $Y$ for every open subset $U$ of $X$, and this means that $f$ is a closed map.

2.3. Proposition. Suppose that $f: X \rightarrow Y$ is a perfect fully closed map onto a connected space $Y$ that contains more than one point. If $f$ is ring-like, then it is atomic.

Proof. It suffices to show that each point-inverse $f^{-1}(y)$ is connected. If the compact space $f^{-1}(y)$ is not connected, there are disjoint open sets $G$ and $H$ of $X$ each intersecting $f^{-1}(y)$ and such that $f^{-1}(y) \subset G \cup H \neq X$. Take open neighborhoods $U, V$ of $y$ with $\operatorname{cl} U, \operatorname{cl} V \subset\{y\} \cup f^{\#}(G) \cup f^{\#}(H), \operatorname{bd} U \subset$ $f^{\#}(G)$, and bd $V \subset f^{\#}(H)$. It is readily seen that $\{y\} \cup\left(f^{\#}(G) \cap V\right)=$ $\operatorname{cl} V \backslash f^{\#}(H)$ and $\{y\} \cup\left(f^{\#}(H) \cap U\right)=\operatorname{cl} U \backslash f^{\#}(G)$. Hence,

$$
\{y\} \cup\left(f^{\#}(G) \cap V\right) \cup\left(f^{\#}(H) \cap U\right) \supset U \cap V \ni y
$$

is a non-trivial clopen subset of the connected space $Y$.

In a compact space quasi-components coincide with components, and this implies the following well-known lemma (cf. [13, Theorem 6.2.24]).

2.4. Lemma. Let $E, F$ be closed subsets of a compact space $X$ such that no subcontinuum of $X$ meets both $E$ and $F$. Then there exist disjoint closed subsets $A, B$ of $X$ such that $E \subset A, F \subset B$ and $X=A \cup B$.

2.5. Proposition. Every atomic, fully closed map $f: X \rightarrow Y$ onto a locally compact space $Y$ is ring-like.

Proof. To show that $f$ is ring-like, consider open neighborhoods $U$ of $x$ in $X$ and $V$ of $y=f(x)$ in $Y$. We can assume that $\operatorname{cl} V$ is compact and $f^{-1}(y) \not \subset U$. As $f$ is fully closed, $U^{y}=\left[U \cap f^{-1}(y)\right] \cup f^{-1} f^{\#}(U) \subset U$ is an open neighborhood of $x$ (see [18, II.1.6(5)]). As $f$ is perfect, $F=$ $f^{-1}(\mathrm{cl} V) \backslash U^{y}$ is compact. As $f$ is atomic, each component of $F$ is contained either in $f^{-1}(y)$ or in $X \backslash f^{-1}(y)$. By Lemma 2.4, there are disjoint closed sets $A, B \subset F$ with $f^{-1}(y) \backslash U \subset A, F \backslash f^{-1}(V) \subset B$, and $F=A \cup B$. Observe that $f(A) \cap f(B)=\emptyset$ and $y \in f(A) \subset V \backslash f(B)$. Now, for any open set $V^{\prime}$ with $f(A) \subset V^{\prime} \subset \operatorname{cl} V^{\prime} \subset V \backslash f(B)$, we have bd $V^{\prime} \subset f^{\#}\left(U^{y}\right)=f^{\#}(U)$, and $f$ is ring-like.

2.6. Corollary. Suppose that $f: X \rightarrow Y$ is a perfect, fully closed map onto a connected, locally compact space $Y$ that contains more than one point. Then $f$ is ring-like iff it is atomic. 
2.7. Remarks. (1) There are several conditions which define fully closed maps in an equivalent way (see [18, Section II.1]). For example, a closed map $f: X \rightarrow Y$ is fully closed iff $f(A) \cap f(B)$ is a discrete subspace of $Y$ whenever $A, B \subset X$ are disjoint closed sets.

(2) If $f$ is a fully closed map from a compact metric space, then only countably many point-inverses under $f$ are non-degenerate (see [18, Proposition II.3.10]). On the other hand, if $f$ is a map from a compact space $X$ onto a metric space, each point-inverse under $f$ is metrizable, and only countably many point-inverses are non-degenerate, then $X$ is metrizable - it is not difficult to exhibit a countable network or even a countable base for $X$.

Consider a map $f: X \rightarrow Y$. For a dimension function $\mathrm{d}$, $\mathrm{d} f$ usually denotes the supremum of the set $\left\{\mathrm{d} f^{-1}(y): y \in Y\right\}$. In this paper, however, it is more convenient to adopt the definition

$$
\mathrm{d} f=\sup \left\{\mathrm{d} f^{-1}(M): M \text { is a discrete closed subset of } Y\right\} .
$$

This clearly agrees with the usual definition if $\mathrm{d}$ is dim, ind, or trind, and if $Y$ is compact. Moreover, if $\mathrm{d}$ is one of Ind, $\operatorname{Ind}_{0}$, trInd or $\operatorname{trInd}_{0}$ and $\mathrm{d} f^{-1}(M)<\infty$ for some discrete subset $M$ of $Y$, then $\mathrm{d} f^{-1}(M)=\mathrm{d} f^{-1}(y)$ for some $y \in M$ (cf. Proposition 7.1.21 of [14]). Thus, the two definitions agree when $\mathrm{d} f<\infty$.

2.8. Theorem (Fedorchuk, see [18, Theorem III.2.4]). If $f$ is a fully closed map from a normal space $X$, then $\operatorname{dim} X \leq \max \{\operatorname{dim} f(X), \operatorname{dim} f\}$.

The advantage of $\operatorname{Ind}_{0}$ over other inductive dimensions lies in the following.

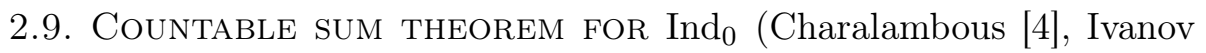
[21]). Suppose that $X=\bigcup_{i=1}^{\infty} F_{i}$ is a normal space, and $F_{i}$ are zero subsets of $X$. If $\operatorname{Ind}_{0} F_{i} \leq n$ for every $i$, then $\operatorname{Ind}_{0} X \leq n$.

The foregoing theorem implies that if $U$ is a cozero subset of a normal space $X$, then $\operatorname{Ind}_{0} U \leq \operatorname{Ind}_{0} X$.

The next three results are proved in [6, Section 2].

2.10. Lemma. Let $\alpha \geq 0$ be an ordinal, $m \geq 0$ an integer, and $E$ a closed subset of a normal space $X$. Suppose that $\operatorname{trnd}_{0} E \leq \alpha$, and $\operatorname{Ind}_{0} F \leq m$ for every closed subset $F$ of $X$ disjoint from $E$. Then $\operatorname{trInd}_{0} X \leq \alpha+m$. If $\alpha$ is a limit ordinal $>0$, then $\operatorname{trInd}_{0} X \leq \alpha$.

2.11. Lemma. Let $E$ be a zero subset of a normal space $X$. Suppose that $A, B$ are disjoint closed subsets of $X$, and $L$ is a zero partition in $E$ between $E \cap A$ and $E \cap B$. Then there are disjoint cozero subsets $G, H$ of $X$ such that $A \subset G, B \subset H, E \backslash L \subset G \cup H$, and cl $G \cap \mathrm{cl} H \subset L$. Furthermore, there is a zero partition $M$ in $X$ between $A$ and $B$ with $M \backslash E \subset X \backslash(\operatorname{cl} G \cup \operatorname{cl} H)$ 
and $M \cap E=L$. If $\operatorname{Ind}_{0}(X \backslash E) \leq m<\infty$, then $M$ can be chosen with $\operatorname{Ind}_{0}(M \backslash L) \leq m-1$.

2.12. Proposition. Let $f: X \rightarrow Y$ be a fully closed map from a nonempty normal space $X$ onto a space $Y$ with $\operatorname{Ind}_{0} Y<\infty$. Suppose that $f(A) \cap f(B)$ is a zero subset of $Y$ for every two disjoint closed subsets $A, B$ of $X$. Then $\operatorname{trInd}_{0} X \leq \operatorname{trInd}_{0} f+\operatorname{Ind}_{0} Y$.

We now turn to the behaviour of Dimensionsgrad under maps. Recall that a space is punctiform if it does not contain non-degenerate continua.

2.13. Lemma. Let $f: X \rightarrow Y$ be a fully closed map from a normal space $X$ onto a punctiform space $Y$. Then $\operatorname{trDg} X=\operatorname{trDg} f$.

Proof. Consider disjoint closed subsets $A, B$ of $X$. Let $M=f(A) \cap f(B)$ and $N=f^{-1}(M) . M$ is discrete by Remark 2.7(1), and $\operatorname{trDg} N \leq \operatorname{trDg} f$. Take a cut $L$ in $N$ between $A \cap N$ and $B \cap N$ with $\operatorname{trDg} L<\operatorname{trDg} f$. Observe that any subcontinuum of $X$ is contained in a single point-inverse of $f$. Hence $L$ is a cut in $X$ between $A$ and $B$, and $\operatorname{trDg} X \leq \operatorname{trDg} f$. That $\operatorname{trDg} X \geq \operatorname{trDg} f$ is evident.

2.14. Proposition. Let $f: X \rightarrow Y$ be a fully closed map from a normal space $X$ onto a space $Y$ with $0 \leq m=\operatorname{Ind}_{0} Y<\infty$. Suppose that $f(A) \cap$ $f(B)$ is a zero subset of $Y$ for every two disjoint closed subsets $A, B$ of $X$. Then $\operatorname{trDg} X \leq \operatorname{trDg} f+\operatorname{Ind}_{0} Y$. If moreover $m>0$, any two disjoint closed subsets of $X$ are separated by a zero partition with $\operatorname{trDg}<\operatorname{trDg} f+\operatorname{Ind}_{0} Y$.

Proof (by induction on $m$ ). By Lemma 2.13, we can assume that $m>0$. Consider disjoint closed subsets $A, B$ of $X$. They are respectively embedded in cozero sets $G, H \subset X$ with $\operatorname{cl} G \cap \operatorname{cl} H=\emptyset$. Because $f$ is fully closed, $M=f(\operatorname{cl} G) \cap f(\operatorname{cl} H)$ is a discrete zero set - see Remark 2.7(1). Then $Z=$ $Y \backslash M$ is a cozero subset of $Y$, and the countable sum theorem for $\operatorname{Ind}_{0}$ implies that $\operatorname{Ind}_{0} Z \leq \operatorname{Ind}_{0} Y=m$. There are disjoint cozero sets $U, V \subset Z$ such that $f(\operatorname{cl} G) \cap Z \subset U, f(\operatorname{cl} H) \cap Z \subset V$, and $\operatorname{Ind}_{0} L \leq m-1$, where $L=Z \backslash(U \cup V)$. Let $K=L \cup M$ and $F=X \backslash\left(f^{-1}(U) \cup f^{-1}(V) \cup G \cup H\right)$. By the countable sum theorem, $\operatorname{Ind}_{0} K \leq m-1$. By the obvious induction hypothesis, $\operatorname{trDg} f^{-1}(K) \leq \operatorname{trDg} f+m-1$. Clearly, the subset $F$ of $f^{-1}(K)$ is a zero partition in $X$ between $A$ and $B$, and $\operatorname{trDg} F \leq \operatorname{trDg} f+m-1$. Hence $\operatorname{trDg} X \leq \operatorname{trDg} f+\operatorname{Ind}_{0} Y$.

2.15. Lemma. Let $X$ be a normal space, and $E \subset X$ a zero subset with $\operatorname{trInd}_{0} E \leq \alpha$ and $\operatorname{trDg}(X \backslash E) \leq \alpha$ (respectively, $\left.\operatorname{trDg}_{0}(X \backslash E) \leq \alpha\right)$. If no subcontinuum of $X$ meets both $E$ and $X \backslash E$, then $\operatorname{trDg} X \leq \alpha$ (respectively, $\left.\operatorname{trDg}_{0} X \leq \alpha\right)$.

Proof (by induction on $\alpha$ ). As the result is evident for $\alpha=-1$, we can suppose $\alpha \geq 0$. Consider disjoint closed subsets $A, B$ of $X$. Let $L$ be a zero 
partition in $E$ between $A \cap E$ and $B \cap E$ with $\operatorname{trInd}_{0} L<\alpha$. By Lemma 2.11, there are disjoint cozero subsets $G, H$ of $X$ such that $A \subset G, B \subset H$ and $\operatorname{cl} G \cap \operatorname{cl} H \subset L$. Let $M$ be a cut (respectively, zero cut) in $X \backslash E$ between $\operatorname{cl} G \backslash E$ and $\operatorname{cl} H \backslash E$ with $\operatorname{trDg} M<\alpha\left(\operatorname{trDg}_{0} M<\alpha\right)$. Evidently $N=L \cup M$ is a closed (zero) subset of $X$. Let $K$ be a subcontinuum of $X$ that meets both $A$ and $B$. Then $K \subset E$ or $K \subset X \backslash E$, and so $K$ meets $L$ or $M$. Thus, $N$ is a cut between $A$ and $B$. By the obvious induction hypothesis, $\operatorname{tr} \operatorname{Dg} N<\alpha$ $\left(\operatorname{trDg}_{0} N<\alpha\right)$. Hence $\operatorname{trDg} X \leq \alpha\left(\operatorname{trDg}_{0} X \leq \alpha\right)$.

3. Resolutions. Suppose we are given a space $X$ and, for each $x \in X$, a map $h_{x}: X \backslash\{x\} \rightarrow Y_{x}$. The resolution space $R=R\left(X, Y_{x}\right)=R\left(X, Y_{x}, h_{x}\right)$ is the set $\bigcup\left\{\{x\} \times Y_{x}: x \in X\right\}$ equipped with the topology generated by all sets of the form

$$
U \otimes_{x} V=\{x\} \times V \cup \bigcup\left\{\{y\} \times Y_{y}: y \in U \cap h_{x}^{-1}(V)\right\},
$$

where $U$ is open in $X, x \in U$, and $V$ is open in $Y_{x}$. The resolution map $\pi: R \rightarrow X$ is the map that sends $(x, y)$ to $x$. If each $Y_{x}$ is compact, then $\pi$ is fully closed. If $X$ and each $Y_{x}$ is compact or first countable, then so is $R$.

Resolutions were introduced by Fedorchuk in [15], and a thorough treatment of fully closed maps and resolutions can be found in [18]. A variant of resolutions for which it is relatively easy to compute inductive dimensions was recently introduced in [6], where all results quoted below can be found.

Let $\mathcal{C}$ be the class of all remainders of compactifications of $[0,1)$. Then $X \in \mathcal{C}$ iff $X$ is a subspace of a compact space $X^{\bullet}$ such that $X^{*}=X^{\bullet} \backslash X$ is a dense subset of $X^{\bullet}$ homeomorphic with $[0,1)$. For every $X \in \mathcal{C}$, we shall assume that some $X^{\bullet}$ has been fixed, and we shall frequently identify the corresponding $X^{*}$ with $[0,1)$. Note that if $X \in \mathcal{C}$, then $X$ is necessarily a continuum, and if $X$ is first countable then so is $X^{\bullet}$. The class $\mathcal{C}$ contains every metric continuum.

Suppose we are given a compact space $X$ and, for each $x \in X$, a space $Y_{x} \in \mathcal{C}$ and a continuous map $h_{x}: X \rightarrow \mathbb{I}$ with $h_{x}^{-1}(1)=\{x\}$ whenever $\{x\}$ is a $G_{\delta}$-subset of $X$. Suppose further that $Y_{x}=\{1\}, Y_{x}^{\bullet}=\mathbb{I}$, and $h_{x}(X)=$ $\{1\}$ whenever $\{x\}$ is not a $G_{\delta}$-subset of $X$. We shall treat the restrictions $h_{x} \mid(X \backslash\{x\})$ as maps into $Y_{x}^{\bullet}$. The resolution space $S=S\left(X, Y_{x}, h_{x}\right)$ is the closed subspace $\bigcup\left\{\{x\} \times Y_{x}: x \in X\right\}$ of $R\left(X, Y_{x}^{\bullet}, h_{x} \mid(X \backslash\{x\})\right)$, and the resolution map $\sigma: S \rightarrow X$ is the function that sends $(x, y)$ to $x$. The topology of $S$ is generated by the sets of the form $\sigma^{-1}(U)$, where $U$ is open in $X$, together with the sets

$$
O(x, V)=\{x\} \times\left(V \cap Y_{x}\right) \cup \sigma^{-1}\left(h_{x}^{-1}(V)\right),
$$

where $\{x\}$ is a $G_{\delta^{-}}$subset of $X, V$ is open in $Y_{x}^{\bullet}$, and $V \cap Y_{x} \neq \emptyset$. 
When $X$ is first countable, the sets $O(x, V)$ form a base for the topology of $S$.

3.1. Proposition. Let $X$ be a compact space. Then $S$ is compact and the resolution map $\sigma: S \rightarrow X$ is monotone, fully closed, and ring-like. If $X$ and each $Y_{x}$ is first countable then so is $S$. If uncountably many $Y_{x}$ contain more than one point, then $S$ is not metrizable.

Suppose that $X$ is a non-degenerate continuum. Then $S$ is a continuum, and it is separable whenever $X$ is. If $X$ and all non-degenerate $Y_{x}$ are hereditarily decomposable or hereditarily indecomposable, then so is $S$. If $X$ is a member of $\mathcal{C}$, then so is $S$.

We usually abbreviate $S\left(X, Y_{x}, h_{x}\right)$ to $S, S(X)$, or $S\left(X, Y_{x}\right)$. If $Y_{x}=Y$ for each $x \in X, S\left(X, Y_{x}, h_{x}\right)$ is denoted by $S_{Y}(X)$, and it is called a standard resolution. Analogous notation and terminology is adopted for $R\left(X, Y_{x}, h_{x}\right)$. Note that $R\left(X, Y_{x}, h_{x}\right)$ is called a ring-like resolution if the corresponding resolution map is ring-like.

The construction of $S\left(X, Y_{x}, h_{x}\right)$ is useful even if $X$ is not first countable. For a metrizable $X$, however, it is possible to choose the maps $h_{x}$ so that $S\left(X, Y_{x}, h_{x}\right)$ acquires additional features.

Let $X$ be a compact metrizable space and, for each $x \in X$, let $Y_{x}$ be a member of $\mathcal{C}$. Let $X^{0}=X \backslash \bigcup\{U \subset X: U$ is open and countable $\}$ be the set of all condensation points of $X$. Then $X \backslash X^{0}$ is clearly countable. If $X$ is uncountable, then $X^{0}$ is a non-empty perfect subset of $X$. Moreover, the collection of all non-empty perfect subsets of $X$ has cardinality $\mathfrak{c}$, and therefore, can be written in the form $\left\{F_{\alpha}: \alpha<\omega(\mathfrak{c})\right\}$ with $\left\{\alpha: F_{\alpha}=X^{0}\right\}$ cofinal in $\omega(\mathfrak{c})$. Equip $X$ with a well-ordering of the same type as $\omega(\mathfrak{c})$ and, for $\alpha<\omega(\mathfrak{c})$, let $x_{\alpha}$ be the first element of $F_{\alpha} \backslash\left\{x_{\beta}: \beta<\alpha\right\}$. Evidently, $x_{\alpha} \neq x_{\beta}$ whenever $\alpha \neq \beta$, and $\left\{x_{\alpha}: \alpha<\omega(\mathfrak{c})\right\}=X^{0}$. There are maps $h_{x_{\alpha}}: X \rightarrow \mathbb{I}$ such that $h_{x_{\alpha}}^{-1}(1)=\left\{x_{\alpha}\right\}$ and $h_{x_{\alpha}}\left(F_{\alpha}\right)=\mathbb{I}$ (cf. [6, Lemma 5]). For all other points $x$ of $X$, we let $h_{x}: X \rightarrow \mathbb{I}$ be any map with $h_{x}^{-1}(1)=\{x\}$. Henceforth, it will be understood that if $X$ is an uncountable compact metric space, the maps $h_{x}$ in the construction of $S\left(X, Y_{x}, h_{x}\right)$ have been chosen as just described. A weak resolution $S\left(X, Y_{x}, h_{x}\right)$ will mean one constructed as described just before Proposition 3.1 .

3.2. Proposition. Let $X$ be a compact metrizable space.

(1) If $F \subset S(X)$ is a closed set such that $\sigma(F)$ is uncountable, then $F$ contains a point-inverse of $\sigma$.

(2) If $X$ is a continuum and $F$ is a partition in $S(X)$, then $F$ contains a point-inverse of $\sigma$.

(3) If $Y_{x}$ is separable for each $x \in X \backslash X^{0}$, then $S(X)$ is separable. 
Proof. If $F \subset S(X)$ is a closed set with $\sigma(F)$ uncountable, then $\sigma(F)$ contains a Cantor set, and $F_{\alpha} \subset \sigma(F)$ for an $\alpha<\omega(\mathfrak{c})$. Now, for every open set $V \subset Y_{x_{\alpha}}^{\bullet}$ that intersects $Y_{x_{\alpha}}, h_{x_{\alpha}}^{-1}(V)$ intersects $F_{\alpha}$ because $h_{x_{\alpha}}\left(F_{\alpha}\right)=\mathbb{I}$. Hence, each $O\left(x_{\alpha}, V\right)$ intersects $F$, and it follows that $\sigma^{-1}\left(x_{\alpha}\right) \subset F$.

For (2) in case $\sigma(F)$ is countable, see [6, the proof of Proposition 7].

Assume that $X$ is uncountable, and $P$ is a countable dense subset of $X^{0}$. For each $x \in X^{0}, h_{x}$ is surjective, and therefore $h_{x}^{-1}(V)$ intersects $P$ whenever $V \subset Y_{x}^{\bullet}$ is an open set with $V \cap Y_{x} \neq \emptyset$. Clearly, $\sigma^{-1}\left(X^{0}\right)$ is separable. If $Y_{x}$ is separable for each $x \notin X^{0}$, then so is $\sigma^{-1}\left(X \backslash X^{0}\right)$, and hence also $S(X)$.

4. Inverse invariance of chainability. A chain is a finite collection of sets $U_{1}, \ldots, U_{n}$ such that $U_{i} \cap U_{j} \neq \emptyset$ iff $|i-j| \leq 1$. A non-empty normal space $X$ is said to be chainable if every open cover of $X$ can be refined by an open (or equivalently, closed) chain. Any chainable space $X$ is clearly a continuum, and $\operatorname{dim} X=1$ unless $X$ is a single point. In this paper, an element $x$ of a chainable space $X$ is called an end point if every open cover of $X$ can be refined by an open (or equivalently closed) chain $\left\{U_{1}, \ldots, U_{n}\right\}$ such that $x \in U_{1}$. In [2] Bing gave an example of a metric chainable continuum without end points.

The pseudo-arc is the only (up to homeomorphism) non-degenerate metric HI chainable continuum (Bing [1]). It will be denoted by $\mathbb{P}$. Since every subcontinuum of a chainable continuum is chainable, every non-degenerate subcontinuum of $\mathbb{P}$ is a pseudo-arc. The pseudo-arc is homogeneous ([1]), and each of its elements is an end point ([2]).

This section contains partial answers to the following general question.

4.1. Problem. Suppose that $f: X \rightarrow Y$ is an atomic, fully closed map, $Y$ is chainable, and every point-inverse of $f$ is chainable. Is $X$ chainable? Under what circumstances is $X$ chainable?

It is interesting to note here the example by Davis and Ingram [12] of a map from a non-chainable atriodic continuum onto a chainable continuum whose unique non-trivial point-inverse is an arc. This map is fully closed, but is not atomic.

4.2. Proposition (cf. [8, Lemma 1], [5, Proposition 3], [6, Proposition 3]). Suppose that $I$ is a linearly ordered continuum. If $f: X \rightarrow I$ is a fully closed atomic map with chainable point-inverses, then $X$ is chainable. If moreover $t$ is an end point of $I$ and $x$ is an end point of $f^{-1}(t)$, then $x$ is an end point of $X$.

Proof. In view of Corollary 2.6, $f$ is ring-like, and $X$ is chainable by [6, Proposition 3]). The assertion on end points is clear from the proof of that result. 
Thus, we have a satisfactory answer to Problem 4.1 if $Y$ is the unit interval. The question naturally arises if in Proposition 4.2 we can replace $I$ with a space such as $\mathbb{P}$. The following is an answer in a very particular case. It will enable us, however, to deduce that certain spaces constructed with the method of resolution are chainable.

4.3. Proposition. Suppose that $X$ is a hereditarily indecomposable continuum, and $T_{1}, \ldots, T_{n} \subset X$ are pairwise disjoint pseudo-arcs. If the map that collapses the continua $T_{1}, \ldots, T_{n}$ to points transforms $X$ onto a pseudoarc, then $X$ is itself a pseudo-arc.

Proof. Let $f: X \rightarrow \mathbb{P}$ be the map that collapses $T_{1}=f^{-1}\left(p_{1}\right), \ldots, T_{n}=$ $f^{-1}\left(p_{n}\right)$ to points $p_{1}, \ldots, p_{n} \in \mathbb{P}$. Then $X$ is metrizable by Remark 2.7 (2).

We apply induction on $n$. Assume $n=1$. Consider the hyperspace $C(X)$ of subcontinua of $X$. It is endowed with the Vietoris topology, which is also induced by the Hausdorff metric $\varrho_{H}$ corresponding to any metric $\varrho$ on $X$. $C(X)$ admits a Whitney map, i.e. a continuous function $\mu: C(X) \rightarrow \mathbb{R}$ such that $(1) \mu(\{x\})=0$ for every $x \in X$, and $(2) \mu(P)<\mu(Q)$ whenever $P \varsubsetneqq Q$ (see [26, pp. 103-109]). As $X$ is an HI continuum, each Whitney level $\mu^{-1}(t), 0 \leq t \leq \mu(X)$, is an upper semicontinuous decomposition of $X$ (see [26, Corollary 1.11.17]). The natural quotient projection $X \rightarrow X / \mu^{-1}(t)$ is moreover open (see [26, Exercise 1.11.23 and its solution, pp. 108 and 538]). Thus, each $\mu^{-1}(t)$ is a continuous decomposition of $X$. The Hausdorff metric $\varrho_{H}$ restricted to $\mu^{-1}(t)$ is compatible with the quotient topology on $X / \mu^{-1}(t)$ (cf. [26, Exercise 1.11.17, p. 108]).

Now, let $t_{0}=\mu\left(T_{1}\right)$ and $\mathcal{D}=\mu^{-1}\left(t_{0}\right)$. Consider the quotient space $X / \mathcal{D}$, and the open, perfect quotient map $q: X \rightarrow X / \mathcal{D}$. As $0<t_{0}<\mu(X), X / \mathcal{D}$ is a non-degenerate metric continuum. Since $q$ is closed and monotone, $X / \mathcal{D}$ is HI. Observe that every continuum $D \in \mathcal{D}$ is a pseudo-arc. Indeed, either $D=T_{1}$, or $D \neq T_{1}$ and the restriction $f \mid D$ is an embedding into $\mathbb{P}$. Since the decomposition $\left\{T_{1}\right\} \cup\left\{\{x\}: x \in X \backslash T_{1}\right\}$ is finer than $\mathcal{D}$, there is a quotient map $g: \mathbb{P} \rightarrow X / \mathcal{D}$ such that $q=g \circ f$. Any point-inverse of $g$ is either $\left\{p_{1}\right\}=f\left(T_{1}\right)$ or $f(D)$, where $T_{1} \neq D \in \mathcal{D}$. Hence, $g$ is a monotone map and $X / \mathcal{D}$ is chainable by a theorem of Bing [1]. Thus, $X$ has a continuous decomposition $\mathcal{D}$ into pseudo-arcs, and $X / \mathcal{D}$ is a pseudo-arc. These two facts imply that $X$ is a pseudo-arc (Lewis [24, Theorem 3]).

Assume that $n>1$. Let $g: X \rightarrow Y$ be the map that collapses only $T_{1}$ to a point, and $h: Y \rightarrow \mathbb{P}$ a map such that $h \circ g=f$. As $g$ is monotone, the continuum $Y$ is HI. Since $h$ collapses pseudo-arcs $g\left(T_{2}\right), \ldots, g\left(T_{n}\right), Y$ and $X$ are pseudo-arcs by the obvious induction hypothesis.

For each ordinal $\alpha>0$, we define a class $\mathcal{P}_{\alpha}$ of (topological types of) non-degenerate continua as follows: 
(a) $X \in \mathcal{P}_{1}$ iff $X$ is a pseudo-arc;

(b) for $\alpha>1, X \in \mathcal{P}_{\alpha}$ iff there exists an atomic fully closed map $f: X \rightarrow \mathbb{P}$ such that for each $p \in \mathbb{P}$ either $f^{-1}(p)$ is a singleton or $f^{-1}(p) \in \mathcal{P}_{\beta}$ for some non-zero ordinal $\beta<\alpha$.

It is clear that $\mathcal{P}_{\alpha} \subset \mathcal{P}_{\beta}$ for $\alpha \leq \beta, \mathcal{P}_{\omega\left(\mathfrak{c}^{+}\right)}=\bigcup_{0<\alpha<\omega\left(\mathfrak{c}^{+}\right)} \mathcal{P}_{\alpha}$, and $\mathcal{P}_{\alpha}=\mathcal{P}_{\omega\left(\mathfrak{c}^{+}\right)}$for $\alpha \geq \omega\left(\mathfrak{c}^{+}\right)$. The map $f$ in the above statement (b) is ring-like by Proposition 2.5, and irreducible by Remark 2.2(1). Using transfinite induction, one easily checks that members of $\mathcal{P}_{\alpha}$ are first countable, separable HI continua.

4.4. Lemma. Suppose that $\alpha>0$ is an ordinal, and $f: X \rightarrow \mathbb{P}$ is an atomic map from a compact space $X$. If $f$ has only finitely many nondegenerate point-inverses each of which belongs to $\mathcal{P}_{\alpha}$, then $X$ is in $\mathcal{P}_{\alpha}$.

Proof. $X$ is an HI continuum since $f$ is atomic. For $\alpha=1$, the only non-degenerate point-inverses of $f$ are pseudo-arcs, and hence the assertion of this lemma is a consequence of Proposition 4.3. Assume $\alpha>1$, and let $T_{1}=f^{-1}\left(p_{1}\right), \ldots, T_{n}=f^{-1}\left(p_{n}\right) \in \mathcal{P}_{\alpha}$ be the only non-degenerate point-inverses. For each $i \in\{1, \ldots, n\}$, there is a fully closed, atomic map $g_{i}: T_{i} \rightarrow \mathbb{P}$ such that each non-degenerate point-inverse $g_{i}^{-1}(p)$ is in $\mathcal{P}_{\beta_{i}^{p}}$, where $\beta_{i}^{p}<\alpha$. Consider the decomposition $\mathcal{D}$ of $X$ into all point-inverses of $g_{i}, i=1, \ldots, n$, and all the remaining single points in $X \backslash\left(T_{1} \cup \cdots \cup T_{n}\right)$, and let $q: X \rightarrow X / \mathcal{D}$ be the quotient projection. It is easily checked that $\mathcal{D}$ is upper semicontinuous, and hence $X / \mathcal{D}$ is a continuum. Evidently, $X / \mathcal{D}$ is HI, and $q$ is fully closed. As $X$ is HI, each $D \in \mathcal{D}$ is a terminal subcontinuum of $X$, and $q$ is atomic. $\mathcal{D}$ is finer than the decomposition of $X$ into point-inverses of $f$, and there is a map $h: X / \mathcal{D} \rightarrow \mathbb{P}$ such that $f=h \circ q$. Clearly, $h$ compresses only the images $q\left(T_{i}\right)$ to points, and each $q\left(T_{i}\right)$ is homeomorphic to $\mathbb{P}$. By Proposition $4.3, X / \mathcal{D}$ is a pseudo-arc. Therefore, $q$ maps $X$ onto a pseudo-arc, and $X \in \mathcal{P}_{\alpha}$.

4.5. TheOREM. Every member of $\mathcal{P}_{\alpha}$ is a chainable continuum all of whose elements are end points.

Proof (by induction on $\alpha$ ). If $\alpha=1$, then $X \in \mathcal{P}_{\alpha}$ is a pseudo-arc, and we are done. Take an ordinal $\alpha>1$, and assume that the theorem is true for each non-zero $\beta<\alpha$ and each continuum in $\mathcal{P}_{\beta}$. Take any $X \in \mathcal{P}_{\alpha}$ and any $x \in X$. Then there is an atomic, fully closed map $f: X \rightarrow \mathbb{P}$ such that for each $p \in \mathbb{P}$, the pre-image $f^{-1}(p)$ is degenerate or belongs to $\mathcal{P}_{\beta^{p}}$ for some $\beta^{p}<\alpha$. In order to prove that $X$ is chainable, take a finite open cover $\mathcal{U}$ of $X$. The set $M=\mathbb{P} \backslash \bigcup\left\{f^{\#}(U): U \in \mathcal{U}\right\}$ is finite (see [18, proof of Theorem III.2.4]). For each $p \in \mathbb{P} \backslash M$, shrink $f^{-1}(p)$ to a point to obtain a space $Y$, a quotient map $g: X \rightarrow Y$, and a map $h: Y \rightarrow \mathbb{P}$ such that $h \circ g=f$. Then $g$ is closed (see the proof of $(5) \Rightarrow(6)$ of the statement II.1.6 in [18]), $Y$ is compact, and $h$ 
is atomic. Lemma 4.4 guarantees that $Y \in \mathcal{P}_{\beta}$, where $\beta=\max \left\{\beta^{p}: p \in M\right\}$ $<\alpha$. The family $\mathcal{V}=\left\{g^{\#}(U): U \in \mathcal{U}\right\}$ is an open cover of $Y$, and by the induction hypothesis, $\mathcal{V}$ has an open chain refinement $\left\{W_{1}, \ldots, W_{n}\right\}$ such that $g(x) \in W_{1}$. Finally, the sets $g^{-1}\left(W_{1}\right) \ni x, g^{-1}\left(W_{2}\right), \ldots, g^{-1}\left(W_{n}\right)$ form an open chain refinement of $\mathcal{U}$.

4.6. Corollary. Every weak standard resolution $S_{\mathbb{P}}(\mathbb{P})$ is a non-metrizable, first countable, separable, hereditarily indecomposable chainable continuum.

4.7. Remark. Chatyrko [7] resolved $\mathbb{P}$ in a manner resembling the construction of the weak $S_{\mathbb{P}}(\mathbb{P})$, obtained a space $B_{1}$, and asked if $B_{1}$ is a chainable continuum with ind $B_{1}=2$. As his resolution map $B_{1} \rightarrow \mathbb{P}$ is atomic and fully closed, and every point-inverse of the map is homeomorphic to $\mathbb{P}, B_{1}$ is certainly chainable. Note that in [6] we have shown that every resolution $S_{\mathbb{P}}(\mathbb{P})$ in the stronger sense has ind $S_{\mathbb{P}}(\mathbb{P})=\operatorname{Ind}_{0} S_{\mathbb{P}}(\mathbb{P})=2$. It will follow from Corollary 5.3 in the present paper that $\operatorname{Dg} S_{\mathbb{P}}(\mathbb{P})=2$, too. In fact, $S_{\mathbb{P}}(\mathbb{P})$ will be the continuum $S_{2}$ of Theorem 5.7.

5. Continua with $\operatorname{dim}<\operatorname{trDg}=\operatorname{trind}=\operatorname{trInd}_{0}$. A space $X$ is said to be non-Suslinian if there exists an uncountable collection of pairwise disjoint non-degenerate subcontinua of $X$.

5.1. Proposition. If $X$ is a non-Suslinian, compact metric space, then there is a point $s \in S(X)$ with an open neighborhood $U$ such that every cut in $S(X)$ between $A=S(X) \backslash U$ and any closed set $B \subset S(X)$ with $s \in \operatorname{int} B \subset B \subset U$ contains a point-inverse of $\sigma: S(X) \rightarrow X$.

Proof. Let $\mathcal{B}$ be a countable base for $X$, and $\mathcal{P}$ an uncountable family of pairwise disjoint non-degenerate subcontinua of $X$. We can assume there is a number $\varepsilon>0$ such that $\operatorname{diam} P>\varepsilon$ for each $P \in \mathcal{P}$. Let $\mathcal{V}=\{V \in \mathcal{B}$ : $V$ meets countably many $P \in \mathcal{P}\}$ and $K=X \backslash \cup \mathcal{V}$. Then the collection $\mathcal{Q}=\{P \in \mathcal{P}: P \subset K\}$ is uncountable, and each open set $V \subset X$ with $V \cap K \neq \emptyset$ meets uncountably many $P \in \mathcal{Q}$. Take $x \in P_{0} \in \mathcal{Q}, s \in \sigma^{-1}(x)$, and put $U=\sigma^{-1}(\mathrm{~B}(x, \varepsilon / 2))$.

Assume that $B \subset U$ is a closed neighborhood of $s$, and $F \subset X$ is a cut in $S(X)$ between $A=S(X) \backslash U$ and $B$. As $\sigma$ is ring-like, there is an open neighborhood $V^{\prime}$ of $x$ such that $\operatorname{cl} V^{\prime} \subset \mathrm{B}(x, \varepsilon / 2)$ and bd $V^{\prime} \subset f^{\#}(\operatorname{int} B)$. Observe that $\sigma(F) \backslash V^{\prime}$ is a cut in $X$ between $X \backslash \mathrm{B}(x, \varepsilon / 2)$ and $\mathrm{cl} V^{\prime}$. Indeed, if $L$ is a subcontinuum of $X$ and $L \backslash \mathrm{B}(x, \varepsilon / 2) \neq \emptyset \neq L \cap \operatorname{cl} V^{\prime}$, then it follows from Lemma 2.4 that a certain component $M$ of $L \backslash V^{\prime}$ meets both $X \backslash \mathrm{B}(x, \varepsilon / 2)$ and bd $V^{\prime}$. So, $\sigma^{-1}(M)$ meets both $A$ and $B, \sigma^{-1}(M)$ meets $F$, and $M \subset L$ meets $\sigma(F) \backslash V^{\prime}$. 
Now, $V^{\prime}$ meets uncountably many $P \in \mathcal{Q}$ which have $\operatorname{diam} P>\varepsilon$, and hence none of them is contained in $\mathrm{B}(x, \varepsilon / 2)$. Therefore, each $P \in \mathcal{Q}$ with $V^{\prime} \cap P \neq \emptyset$ meets the cut $\sigma(F) \backslash V^{\prime}$. Thus, $\sigma(F)$ is uncountable, and $F$ contains a point-inverse of $\sigma$ by Proposition 3.2(1).

5.2. Corollary. Suppose that $X$ is a non-Suslinian, compact metric space, and $\operatorname{trDg} Y_{x} \geq \alpha$ (respectively, trind $\left.Y_{x} \geq \alpha\right)$ for each $x \in X$. Then $\operatorname{trDg} S\left(X, Y_{x}\right) \geq \alpha+1$ (respectively, trind $\left.S\left(X, Y_{x}\right) \geq \alpha+1\right)$.

Using the foregoing corollary and Proposition 2.14, we obtain

5.3. Corollary. If $X$ is a non-Suslinian, one-dimensional, compact metric space, and $Y \in \mathcal{C}$, then $\operatorname{trDg} S_{Y}(X)=\operatorname{trDg} Y+1$.

5.4. Remark. The statements 5.15.3 also hold true for some Suslinian spaces $X$. Mazurkiewicz 25] constructed a locally connected, metric Suslinian (hence, one-dimensional) continuum $X$ which is not rim-countable at any point. Thus, each point $x \in X$ has an open neighborhood $V_{x}$ such that every partition in $X$ between $X \backslash V_{x}$ and $\{x\}$ is uncountable. As is easily seen, in locally connected Polish spaces the notions of a cut and of a partition are equivalent (cf. [13, Problems 6.3.3(a) and 6.3.11]). The proof of Proposition 5.1 works because, when we replace $\mathrm{B}(x, \varepsilon / 2)$ with $V_{x}$, the cut $\sigma(F) \backslash V^{\prime}$ in $X$ is a partition between $A=X \backslash V_{x}$ and $\mathrm{cl} V^{\prime}$, and hence it is uncountable.

5.5. Proposition. Suppose that $f: X \rightarrow Y$ is a closed, monotone ringlike map onto a $\sigma$-compact metric space $Y$ with $\operatorname{dim} Y \geq n>0$. Let

$$
\mu=\mu(Y)=\min \left\{\operatorname{trDg} f^{-1}(L): L \subset Y \text { is a non-degenerate continuum }\right\} .
$$

Then $\operatorname{trDg} X \geq \mu+n-1$.

Proof (by induction on $n$ ). By the countable sum theorem (see [14, Theorems 1.5.3 and 1.7.7]), $Y$ contains a compact subspace with $\operatorname{dim} \geq n$. We can therefore assume that $Y$ is compact. Note that, as $f$ is monotone, $f^{-1}(L)$ is a continuum for every continuum $L \subset Y$. Thus, $\mu \geq 1$. For $n=1$, choosing $L$ with $\mu=\operatorname{trDg} f^{-1}(L)$, we obtain $\operatorname{trDg} X \geq \operatorname{trDg} f^{-1}(L)=$ $\mu+n-1$. Assume that $n>1$ and that the result holds for smaller integers $\geq 1$. Let $A, B \subset Y$ be disjoint closed sets such that every cut in $Y$ between $A$ and $B$ has $\operatorname{Dg}=\operatorname{dim} \geq n-1$. If $D$ is a cut between $f^{-1}(A)$ and $f^{-1}(B)$, then $f(D)$ is a cut between $A$ and $B$ by Proposition 2.1. Hence, $\operatorname{dim} f(D)=\operatorname{Dg} f(D) \geq n-1$. Now, $f(D)$ contains a connected component $K$ such that $\operatorname{dim} K \geq n-1$ and, because $f$ is ring-like, $f^{-1}(K) \subset D$. Applying the induction hypothesis to $f \mid f^{-1}(K): f^{-1}(K) \rightarrow K$, we obtain $\operatorname{trDg} D \geq \operatorname{trDg} f^{-1}(K) \geq \mu(K)+n-2 \geq \mu+n-2$. Hence $\operatorname{trDg} X \geq$ $\mu+n-1$. 
5.6. Corollary. Let $X$ be a compact metric space with $0<\operatorname{dim} X<\infty$ and $Y \in \mathcal{C}$. Then

$$
\operatorname{trDg} Y+\operatorname{dim} X-1 \leq \operatorname{trDg} S_{Y}(X) \leq \operatorname{trDg} Y+\operatorname{dim} X
$$

even for the weak version of $S_{Y}(X)$. If $X$ is additionally hereditarily indecomposable, and $S_{Y}(X)$ is a resolution in the stronger sense, then

$$
\operatorname{trDg} S_{Y}(X)=\operatorname{trDg} Y+\operatorname{dim} X .
$$

Proof. That $\operatorname{trDg} S_{Y}(X) \leq \operatorname{trDg} Y+\operatorname{dim} X$ follows from Proposition 2.14. If $L$ is a non-degenerate subcontinuum of $X$, then $\sigma^{-1}(L)$ contains a copy of $Y$. Consequently, $\mu(X) \geq \operatorname{trDg} Y$. If $X$ is $\mathrm{HI}$ and $S_{Y}(X)$ is a resolution in the stronger sense, then $L$ is non-Suslinian, $\operatorname{trDg} \sigma^{-1}(L) \geq$ $\operatorname{trDg} Y+1$ by Corollary 5.2, and $\mu(X) \geq \operatorname{trDg} Y+1$.

5.7. Theorem. For every ordinal $\alpha>0$ of cardinality $\leq \mathfrak{c}$ there exists a separable, first countable, chainable, hereditarily indecomposable continuum $S_{\alpha} \in \mathcal{C} \cap \mathcal{P}_{\alpha}$ such that $\operatorname{trDg} S_{\alpha}=\operatorname{trind} S_{\alpha}=\operatorname{trInd}_{0} S_{\alpha}=\alpha$ and each element in $S_{\alpha}$ is an end point.

Proof (by induction on $\alpha$ ). We put $S_{\alpha}=\mathbb{P}$ for $\alpha=1$. Assuming $S_{\alpha}$ has been constructed, we put $S_{\alpha+1}=S_{S_{\alpha}}(\mathbb{P}) \in \mathcal{P}_{\alpha+1}$. By Proposition 3.1, $S_{\alpha+1}$ is a separable, first countable HI member of $\mathcal{C}$. Theorem 4.5 implies that $S_{\alpha+1}$ is chainable and each of its elements is an end point. Corollary 5.2 yields $\alpha+1 \leq \operatorname{trDg} S_{\alpha+1}$ and $\alpha+1 \leq \operatorname{trind} S_{\alpha+1}$. By Proposition 2.12, $\operatorname{trInd}_{0} S_{\alpha+1} \leq \alpha+1$, and hence all three inductive dimension functions are equal to $\alpha+1$ for $S_{\alpha+1}$.

Let $\alpha$ be a limit ordinal, and assume that $S_{\beta}$ have been constructed for each $\beta<\alpha$. Take a Cantor set $F \subset \mathbb{P}$, and choose distinct points $x_{\beta} \in F$ for $\beta<\alpha$. We put $Y_{x_{\beta}}=S_{\beta}$ for $\beta<\alpha$, and $Y_{x}$ are singletons for other points $x \in \mathbb{P}$. Then $S_{\alpha}=S\left(\mathbb{P}, Y_{x}\right) \in \mathcal{P}_{\alpha}$ obviously satisfies $\alpha \leq \operatorname{trDg} S_{\alpha}$ and $\alpha \leq \operatorname{trind} S_{\alpha}$. We obtain $\operatorname{trInd}_{0} \sigma^{-1}(F) \leq \alpha$ by Proposition 2.12, and $\operatorname{trInd}_{0} S_{\alpha} \leq \alpha$ by Lemma 2.10. Finally, Theorem 4.5 yields chainability with the end point property.

5.8. Remarks. (1) It is worth recalling here that any first countable compact space $X$ has cardinality $\leq \mathfrak{c}$ (see [13, Corollary 3.1.30]), and hence if $X$ has trind $X<\infty$ (respectively, trInd $X<\infty$ ), then also the cardinality of trind $X$ (respectively, trInd $X$ ) is $\leq \mathfrak{c}$ (see [14, Theorems 7.1.6 and 7.1.17]).

(2) Chatyrko and Fedorchuk [1] asked whether $\operatorname{Dg} X=1$ for every non-degenerate chainable continuum $X$ (see also Chatyrko [9, Question 6] in Open Problems in Topology II). Thus, Theorem 5.7 is a negative answer to their question.

(3) Theorem 5.7 provides a positive answer in the realm of first countable spaces to Pasynkov's question [27] whether for every ordinal $\alpha$, there exists 
a chainable continuum with trind $=\alpha$. Note that in [6] we have constructed a first countable, separable, HD chainable continuum $S_{\alpha}$ with trind $S_{\alpha}=$ $\operatorname{trInd}_{0} S_{\alpha}=\alpha$, for each $\alpha$ of cardinality $\leq \mathfrak{c}$.

5.9. TheOREM. For each natural number $n \geq 1$ and each ordinal $\alpha \geq n$ of cardinality $\leq \mathfrak{c}$ there exists a separable, first countable, hereditarily indecomposable continuum $S_{n, \alpha} \in \mathcal{C}$ such that $\operatorname{dim} S_{n, \alpha}=n$ and $\operatorname{trDg} S_{n, \alpha}=$ trind $S_{n, \alpha}=\operatorname{trInd}_{0} S_{n, \alpha}=\alpha$.

Proof. By a theorem of Bing, there is a metric HI continuum $S_{n, n}$ with $\operatorname{dim} S_{n, n}=n$ (see van Mill [26, Corollary 3.8.3]). For $\alpha>n$ we construct $S_{n, \alpha}$ in the same way as we constructed $S_{\alpha}$ in the proof of Theorem 5.7, setting $S_{n, \alpha+1}=S_{S_{n, \alpha}}(\mathbb{P})$, and in the case of a limit ordinal $\alpha$, letting $Y_{x_{\beta}}=S_{n, \beta}$ for $\beta<\alpha$. The inequality $\operatorname{dim} S_{n, \alpha} \geq n$ is obvious, and that $\operatorname{dim} S_{n, \alpha} \leq n$ follows from Theorem 2.8 .

To construct our next series of examples, we shall use the Cantor fan $\mathbb{J} \subset \mathbb{R}^{2}$. It is the union of all segments with endpoints $(0,1),(c, 0) \in \mathbb{R}^{2}$, where $c$ are elements of the ternary Cantor set $C \subset \mathbb{I}$. $\mathbb{J}$ is obviously nonSuslinian.

5.10. TheOREM. For every ordinal $\alpha>0$ of cardinality $\leq \mathfrak{c}$ there exists a separable, first countable, hereditarily decomposable continuum $S_{\alpha} \in \mathcal{C}$ such that $\operatorname{dim} S_{\alpha}=1$ and $\operatorname{trDg} S_{\alpha}=\operatorname{trind} S_{\alpha}=\operatorname{tr} \operatorname{Ind}_{0} S_{\alpha}=\alpha$.

Proof. We construct $S_{\alpha}$ as in the proof of Theorem 5.7, but we use the Cantor fan $\mathbb{J}$ instead of $\mathbb{P}$. We obtain $\operatorname{dim} S_{\alpha} \leq 1$ by Theorem 2.8 .

5.11. COROLlary. There is a hereditarily decomposable continuum (respectively, a chainable continuum with an end point) $S_{\omega\left(\mathfrak{c}^{+}\right)}$such that $\operatorname{dim} S_{\omega\left(\mathfrak{c}^{+}\right)}=1$ and $\operatorname{trDg} S_{\omega\left(\mathfrak{c}^{+}\right)}=\operatorname{trind} S_{\omega\left(\mathfrak{c}^{+}\right)}=\operatorname{tr} \operatorname{Ind}_{0} S_{\omega\left(\mathfrak{c}^{+}\right)}=\omega\left(\mathfrak{c}^{+}\right)$.

Proof. Consider the long segment $L$ of length $\alpha=\omega\left(\mathfrak{c}^{+}\right)$(cf. [13, Problem 3.12.19]). Let $W$ and $W_{1}$ be, respectively, the sets of all ordinals and all non-limit ordinals $\leq \alpha$ in $L$. Each $\beta \in W_{1}$ is a $G_{\delta}$-point of $L$, and we let $Y_{\beta}$ be the space $S_{\beta} \in \mathcal{C}$ of Theorem 5.10 (respectively, 5.7). For all other points $x$ of $L$, we let $Y_{x}=\{1\}$. Let $S_{\alpha}$ be a weak resolution $S\left(L, Y_{x}\right)$. Then $S_{\alpha}$ is an HD continuum (respectively, a chainable continuum with an end point) by Proposition 3.1 (respectively, 4.2). It is clear that $\operatorname{dim} S_{\alpha}=1$, $\operatorname{trDg} S_{\alpha} \geq \alpha$ and trind $S_{\alpha} \geq \alpha$. For the inequality $\operatorname{trInd}_{0} S_{\alpha} \leq \alpha$, see [6, proof of Corollary 5].

6. Extending cuts. The main result of this section is a lemma on extending zero cuts. It is an indispensable tool for proving the two theorems of this section as well as other results that are needed for computing the Dimensionsgrad of continua in our final section. 
6.1. Lemma. Let $X$ be a compact space, $A, B \subset X$ disjoint closed sets, and $X_{1}, X_{2}, \ldots, X_{n}, \ldots \subset X$ pairwise disjoint open subcontinua. If each $X_{n}$ meets both $A$ and $B$, then so does a certain subcontinuum of $X \backslash \bigcup_{n \in \mathbb{N}} X_{n}$.

Proof. If no subcontinuum of $X_{0}=X \backslash \bigcup_{n \in \mathbb{N}} X_{n}$ meets both $A$ and $B$, then by Lemma 2.4, there are disjoint closed sets $E, F \subset X_{0}$ such that $A \cap X_{0} \subset E, B \cap X_{0} \subset F$, and $X_{0}=E \cup F$. Then there are disjoint open sets $U, V \subset X$ such that $A \cup E \subset U$ and $B \cup F \subset V$. Since $X=U \cup V \cup \bigcup_{n \in \mathbb{N}} X_{n}$ is compact, a certain $X_{n}$ is contained in $U \cup V$, contradicting the fact that $X_{n}$ is connected.

6.2. Lemma. Let $f: X \rightarrow Y$ be an atomic, fully closed map onto a locally compact and collectionwise Hausdorff space $Y$. Let $A, B \subset X$ be disjoint closed sets with $S=f(A) \cap f(B)$ a zero subset of $Y$. Let $E$ be a cut in $T=f^{-1}(S)$ between $A \cap T$ and $B \cap T, V \subset X$ an open set with $E \subset V \subset$ $X \backslash(A \cup B)$, and $W \subset Y$ an open neighborhood of $S$. Then there is an open neighborhood $W^{\prime}$ of $S$ such that $\mathrm{cl} W^{\prime} \subset W$ is the sum of compact subsets of $Y$, bd $W^{\prime} \subset f^{\#}(V)$, and for every continuum $K \subset X \backslash f^{-1}\left(S \cup f^{\#}(V)\right)$ that meets both $A$ and $B, f(K)$ is disjoint from $\mathrm{cl} W^{\prime}$.

Proof. Since $f$ is fully closed, the set $(V \cap T) \cup f^{-1}\left(f^{\#}(V)\right)$ is open in $X$ (cf. [18, II.1.6]). So, we can assume that $V \backslash T=f^{-1}\left(f^{\#}(V)\right)$. As $S$ is a discrete set and $Y$ is locally compact and collectionwise Hausdorff, there is a discrete collection $\left\{G_{s}: s \in S\right\}$ of open subsets of $Y$ with $s \in G_{s}$ and $\operatorname{cl} G_{s} \subset W$ compact. It therefore suffices to find a collection $\left\{W_{s}: s \in S\right\}$ of open sets $W_{s}$ such that $s \in W_{s} \subset G_{s}$, bd $W_{s} \subset f^{\#}(V)$, and for every continuum $K \subset X \backslash f^{-1}\left(S \cup f^{\#}(V)\right)$ that meets both $A$ and $B, f(K)$ is disjoint from $\mathrm{cl} W_{s}$.

Write $\{s\}=\bigcap_{n \in \mathbb{N}} W_{n}$, where $W_{n}$ is open and $\mathrm{cl} W_{n+1} \subset W_{n} \subset G_{s}$. Since $f$ is ring-like by Proposition 2.5, we can assume that bd $W_{n} \subset f^{\#}(V)$. If $K \subset X \backslash f^{-1}\left(S \cup f^{\#}(V)\right)$ is a continuum, then either $f(K) \cap \mathrm{cl} W_{1}=\emptyset$ or $f(K)$ is contained in some $W_{n} \backslash \mathrm{cl} W_{n+1}$. If there is no $W_{s}$ with the properties listed in the previous paragraph, we can assume that for each $n$ there is a continuum $K_{n} \subset f^{-1}\left(W_{n} \backslash \operatorname{cl} W_{n+1}\right) \backslash f^{-1}\left(f^{\#}(V)\right)=f^{-1}\left(W_{n} \backslash \operatorname{cl} W_{n+1}\right) \backslash V$ that meets both $A$ and $B$. Now, it follows from Lemma 6.1 that $T \cap c 1\left(\bigcup_{n \in \mathbb{N}} K_{n}\right)$ contains a continuum $L$ that meets both $A$ and $B$. Therefore $L$ meets $E$, and $V$ meets some $K_{n}$. This contradiction proves the lemma.

Suppose that $X$ is a space and $\mathcal{F}$ is a family of closed subspaces of $X$. Then $\mathcal{F}$ is called a strong cut (partition) system for $X$ if $\emptyset \in \mathcal{F}$ and for every pair of disjoint closed sets $A, B \subset X$ there is a cut (partition) $F \in \mathcal{F}$ in $X$ between $A$ and $B$.

6.3. Lemma on extending Cuts. Let $f: X \rightarrow Y$ be an atomic, fully closed map onto a locally compact and collectionwise Hausdorff space $Y$. Let 
$A, B \subset X$ be disjoint closed sets with $S=f(A) \cap f(B)$ a zero subset of $Y$. Suppose that $E$ is a zero cut in $T=f^{-1}(S)$ between $A \cap T$ and $B \cap T$, and $\mathcal{F}$ is a strong system of zero cuts for $Y$. Then, for each $n \in \mathbb{N}$, there is $F_{n} \in \mathcal{F}$ with $F_{n} \subset Y \backslash S$ such that the family $\left\{F_{n}: n \in \mathbb{N}\right\}$ is discrete in $Y \backslash S$ and $E \cup \bigcup_{n \in \mathbb{N}} f^{-1}\left(F_{n}\right)$ constitutes a zero cut in $X$ between $A$ and $B$.

Proof. For $n=1,2, \ldots$, there are cozero subsets $V_{n}$ and zero subsets $Z_{n}$ of $X$ with cl $V_{n+1} \subset Z_{n} \subset V_{n} \subset X \backslash(A \cup B)$ and $E=\bigcap_{n \in \mathbb{N}} V_{n}=\bigcap_{n \in \mathbb{N}} Z_{n}$. Applying Lemma 6.2, we obtain open sets $W_{n} \subset Y$ such that $\operatorname{cl} W_{n+1} \subset W_{n}$, $\operatorname{cl} W_{1}$ is the sum of compact subspaces of $Y$, bd $W_{n} \subset f^{\#}\left(V_{n}\right), S=\bigcap_{n \in \mathbb{N}} W_{n}$, and for every continuum $K \subset X \backslash f^{-1}\left(S \cup f^{\#}\left(V_{n}\right)\right)$ that meets both $A$ and $B$, $f(K)$ is disjoint from $\mathrm{cl} W_{n}$. Note that bd $W_{n}$ is disjoint from both $f(A)$ and $f(B)$. Let $F_{1} \in \mathcal{F}$ be a cut between $f(A) \cup \operatorname{cl} W_{1}$ and $f(B) \backslash W_{1}$. For $n>1$, let $J_{n}=\operatorname{cl} W_{n-1} \backslash\left(W_{n} \cup f^{\#}\left(V_{n-1}\right)\right) \subset W_{n-1} \backslash \operatorname{cl} W_{n}$. Observe that $J_{n}$ is the sum of compact spaces, and no component $L$ of $J_{n}$ meets both $f(A)$ and $f(B)-$ otherwise the continuum $K=f^{-1}(L) \subset X \backslash f^{-1}\left(S \cup f^{\#}\left(V_{n-1}\right)\right)$ would meet both $A$ and $B$. By Lemma 2.4, there are disjoint closed sets $A_{n}, B_{n} \subset Y$ such that $f(A) \cap J_{n} \subset A_{n}, f(B) \cap J_{n} \subset B_{n}$, and $J_{n}=A_{n} \cup B_{n}$. Now, $A_{n}^{\prime}=\left(Y \backslash W_{n-1}\right) \cup \mathrm{cl} W_{n} \cup A_{n}$ and $B_{n}$ are disjoint and closed subsets of $Y$. Let $F_{n} \in \mathcal{F}$ be a cut between $A_{n}^{\prime}$ and $B_{n}$. Then $F_{n} \subset f^{\#}\left(V_{n-1}\right) \cap\left(W_{n-1} \backslash \operatorname{cl} W_{n}\right)$ for $n>1$, and $F_{1} \cap \operatorname{cl} W_{1}=\emptyset$. It follows that the family $\left\{F_{n}: n \in \mathbb{N}\right\}$ is discrete in $Y \backslash S$, the set $F=E \cup \bigcup_{n \in \mathbb{N}} f^{-1}\left(F_{n}\right)$ is disjoint from $A \cup B$, and $F=\bigcap_{n \in \mathbb{N}}\left[Z_{n} \cup \bigcup_{m=1}^{n+1} f^{-1}\left(F_{m}\right)\right]$. As each $Z_{n} \cup \bigcup_{m=1}^{n+1} f^{-1}\left(F_{m}\right)$ is a zero subset of $X$, so is $F$.

Let $K \subset X$ be a continuum which meets both $A$ and $B$. It remains to show that $K$ meets $F$. This is clear if $K$ meets $T$, and we can suppose $K \cap T=\emptyset$ and $K=f^{-1}(f(K))$. If $f(K)$ meets $f(B)$ only outside $W_{1}$, then $f(K)$ meets $F_{1}$ and $K$ meets $f^{-1}\left(F_{1}\right) \subset F$. Thus, we can assume that for some $n_{0}>1, f(K)$ meets $f(B) \cap J_{n_{0}}$. Now, if $f(K)$ does not meet $f(A) \cap J_{n_{0}}$, then it meets bd $W_{n_{0}-1}$ or bd $W_{n_{0}}$. In any case, $f(K)$ meets both $A_{n_{0}}^{\prime}$ and $B_{n_{0}}$. Hence, $f(K)$ meets $F_{n_{0}}$ and $K$ meets $f^{-1}\left(F_{n_{0}}\right) \subset F$. Thus, $F$ is a zero cut between $A$ and $B$.

6.4. Lemma. If $Y$ is a rim-finite, paracompact, locally compact space, then the family of all closed discrete subsets constitutes a strong partition system for $Y$.

Proof. Consider disjoint closed sets $A, B$ of $Y$. Then $Y$ has a locally finite open cover $\left\{V_{i}: i \in I\right\}$, where each $\mathrm{cl} V_{i}$ is compact with either $A \cap \operatorname{cl} V_{i}=\emptyset$ or $B \cap \operatorname{cl} V_{i}=\emptyset$. Take a closed cover $\left\{F_{i}: i \in I\right\}$ of $Y$ with $F_{i} \subset V_{i}$. As each $F_{i}$ is compact, there is an open set $U_{i}$ with $F_{i} \subset U_{i} \subset \operatorname{cl} U_{i} \subset V_{i}$ and bd $U_{i}$ finite. Let $U=\bigcup\left\{U_{i}: U_{i} \cap A \neq \emptyset\right\}$. Then the discrete set bd $U \subset$ $\bigcup\left\{\operatorname{bd} U_{i}: U_{i} \cap A \neq \emptyset\right\}$ is a partition between $A$ and $B$. 
For the next result, it is useful to recall that in a normal space a zero set is simply a closed $G_{\delta}$-set. Also, paracompactness implies collectionwise normality, and paracompactness is an inverse invariant under perfect maps [13].

6.5. Theorem. Let $f: X \rightarrow Y$ be an atomic, fully closed map onto a rim-finite, first countable, locally compact, paracompact space $Y$. Suppose that every point-inverse of $f$ contains an element of countable character. Then $\operatorname{trDg}_{0} X \leq \max \left\{1, \operatorname{trDg}_{0} f\right\}$.

Proof. Since $Y$ is collectionwise normal and first countable, every discrete closed subspace of $Y$ is a $G_{\delta}$-set, and hence a zero subset of $Y$. By Lemma 6.4. the family $\mathcal{F}$ of all discrete closed subsets of $Y$ is a strong partition system of zero sets for $Y$. Hence $\operatorname{trDg}_{0} Y \leq 1$. If $\operatorname{trDg}_{0} f=0$, then $f$ is a homeomorphism and the result clearly holds. Assume that $\alpha=\operatorname{trDg}_{0} f \geq 1$. Consider disjoint non-empty closed sets $A, B \subset X$. Since $f$ is fully closed, $S=f(A) \cap f(B)$ is discrete, and hence it is a zero subset of $Y$. Then $T=f^{-1}(S)$ has $\operatorname{trDg}_{0} T \leq \alpha$, and there is a zero cut $E$ in $T$ between $A \cap T$ and $B \cap T$, where $\operatorname{trDg}_{0} E<\alpha$. By the lemma on extending cuts, $E$ is contained in a zero cut $F$ in $X$ between $A$ and $B$, where $F \backslash E=$ $\bigcup_{n \in \mathbb{N}} f^{-1}\left(F_{n}\right), F_{n} \in \mathcal{F}$ for each $n$, and the family $\left\{F_{n}: n \in \mathbb{N}\right\}$ is discrete in $Y \backslash S$. Note that the last property implies that each $f^{-1}\left(F_{n}\right)$ is clopen in $F$. For each $y \in F_{n}$, let $x_{y} \in f^{-1}(y)$ be a point of countable character. Put $G=E \cup\left\{x_{y}: y \in F_{n}, n \in \mathbb{N}\right\}$. For each $n$, the set $\left\{x_{y}: y \in F_{n}\right\}$ is a zero set of the normal space $X$, and so is $\left(F \backslash f^{-1}\left(F_{n}\right)\right) \cup\left\{x_{y}: y \in F_{n}\right\}$. Hence $G$ is a zero set as the intersection of countably many zero sets.

Take a continuum $K \subset X$ that meets both $A$ and $B$. As $F$ is a cut between $A$ and $B$, there is a point $x_{0} \in K \cap F$. If $x_{0} \in E$, then $K$ meets $G$. If $x_{0} \notin E$, we have $f\left(x_{0}\right) \in F_{n_{0}}$ for a certain $n_{0}$. Then $K=f^{-1} f(K)$, and $x_{f\left(x_{0}\right)} \in f^{-1}\left(f\left(x_{0}\right)\right) \cap G \subset K \cap G$. Thus, in any case $K$ meets $G$, and $G$ is a zero cut between $A$ and $B$. Since each non-degenerate subcontinuum of $G$ is contained in $E$, we have $\operatorname{trDg}_{0} G=\operatorname{trDg}_{0} E<\alpha$. We have shown that $\operatorname{trDg}_{0} X \leq \alpha$.

By an obvious modification of the foregoing proof, we obtain

6.6. Theorem. Let $f: X \rightarrow Y$ be an atomic fully closed map onto a non-degenerate linearly ordered continuum $Y$ that contains a dense subset of points of countable character. Suppose that every non-degenerate pointinverse of $f$ is a $G_{\delta}$-subset of $X$ and contains an element of countable character. Then $\operatorname{trDg}_{0} X=\max \left\{1, \operatorname{trDg}_{0} f\right\}$.

It is interesting to look for other classes of one-dimensional compact metric spaces $Y$ for which the inequality $\operatorname{trDg} \operatorname{Dg}_{0} X \leq \max \left\{1, \operatorname{trDg}_{0} f\right\}$ holds for any atomic, fully closed map $f: X \rightarrow Y$ from a first countable space $X$. One such space is $Y=\mathbb{S} \times \mathbb{I}$, where $\mathbb{S}$ stands for $\{0\} \cup\{1 / n: n \in \mathbb{N}\}$. 
Note that the inequality fails if $Y$ is the Cantor fan $\mathbb{J}$ (by Corollary 5.2 or Mazurkiewicz's Suslinian continuum of Remark 5.4.

6.7. Corollary. If $f: X \rightarrow \mathbb{S} \times \mathbb{I}$ is an atomic, fully closed map from a first countable space $X$, then $\operatorname{trDg}_{0} X=\max \left\{1, \operatorname{trDg}_{0} f\right\}$.

Proof. Write $\alpha=\max \left\{1, \operatorname{trDg}_{0} f\right\}$, and consider the map $g: \mathbb{S} \times \mathbb{I} \rightarrow Y$ that collapses the segment $\{0\} \times \mathbb{I}$ to a point. Observe that the composition $h=g \circ f$ is an atomic fully closed map. Indeed, if $A, B \subset X$ are disjoint closed sets, then $f(A) \cap f(B)$ is finite and $h(A) \cap h(B) \subset g(f(A) \cap f(B)) \cup$ $\{g(0,0)\}$-cf. Remark 2.7 (1). It is clear that $T=f^{-1}(\{0\} \times \mathbb{I})$ is a terminal subcontinuum of $X$, and $h$ is atomic. By Theorem 6.5. we have $\operatorname{trDg}_{0} T \leq \alpha$, and hence $\operatorname{trDg}_{0} h=\alpha$. As $Y$ is rim-finite, we again obtain $\operatorname{trDg} \operatorname{gg}_{0} X=$ $\max \left\{1, \operatorname{trDg}_{0} h\right\}=\alpha$.

Given a dimension function $\mathrm{d}$ and a class $\mathcal{M}$ of maps, one says that the theorem on dimension lowering maps holds in $\mathcal{M}$ if $\mathrm{d} X \leq \mathrm{d} Y+\mathrm{d} f$ for each map $f: X \rightarrow Y$ in $\mathcal{M}$ (cf. [14, Theorems 1.12.4, 3.3.10, and 4.3.4]). We end this section with an example which shows that the theorem on dimensionlowering maps does not hold for Dimensionsgrad and maps between compact spaces. This is no surprise because there are analogous counter-examples also for ind, Ind, and Ind $_{0}$ (see Chatyrko [10], Krzempek [23]).

6.8. ExAmple. Let $C$ be a Cantor set, and consider the first countable compact space $X=S_{\mathbb{I}}(C \times \mathbb{I})$. The space is separable by Proposition 3.2. (3). We have $\operatorname{Dg} X=\operatorname{Dg}_{0} X=\operatorname{ind} X=\operatorname{Ind}_{0} X=2$ by Corollary 5.2 and Proposition 2.12. Each component $P$ of $X$ has $\operatorname{Dg} P=\operatorname{Dg}_{0} P=1$ by Theorem 6.5. and Proposition 3.2(2) implies that ind $P=\operatorname{Ind}_{0} P=2$. Let us consider the map $f: X \rightarrow C$ which collapses each component of $X$ to a point. Then $\operatorname{Dg} X=\operatorname{Dg}_{0} X=2>1=\operatorname{Dg} C+\operatorname{Dg} f=\operatorname{Dg}_{0} C+\operatorname{Dg}_{0} f$.

7. Continua with $\operatorname{trDg}<\operatorname{trind}=\operatorname{trInd}_{0}$. In this section we present the second part of the main results of this paper.

7.1. Remark. In [5] Charalambous constructed a sequence of chainable spaces $X_{n}, n=1,2, \ldots$, and showed that $1 \leq \operatorname{Dg} X_{n}<\operatorname{ind} X_{n}=$ $\operatorname{Ind}_{0} X_{n}=n$ for $n>1$. The space $X_{n}$ is closely related to the space $S_{n}$ of Theorem 7.2. More precisely: $X_{1}=\mathbb{I}, X_{n+1}$ is obtained by resolving $\mathbb{I}$ at each point $x \in(0,1)$ into $X_{n}$, and the resolution map $X_{n+1} \rightarrow \mathbb{I}$ is atomic and fully closed. It therefore follows from Theorem 6.5 that, in fact, $\operatorname{Dg} X_{n}=\operatorname{Dg}_{0} X_{n}=1$.

7.2. TheOrem. For every ordinal $\alpha>0$ of cardinality $\leq \mathfrak{c}$ there exists a chainable, separable, first countable, hereditarily decomposable continuum $S_{\alpha} \in \mathcal{C}$ such that $\operatorname{Dg} S_{\alpha}=\operatorname{Dg}_{0} S_{\alpha}=1$, trind $S_{\alpha}=\operatorname{trInd}_{0} S_{\alpha}=\alpha$, and $S_{\alpha}$ has an end point. 
Proof. We construct $S_{\alpha}$ as in the proof of Theorem 5.7, using II instead of $\mathbb{P}$. Briefly, for non-limit ordinals, $S_{1}=\mathbb{I}$ and $S_{\alpha+1}=S_{S_{\alpha}}(\mathbb{I})$. For a limit ordinal $\alpha$, we choose distinct points $x_{\beta}, \beta<\alpha$, belonging to a Cantor set $F \subset \mathbb{I}$ and set $S_{\alpha}=S\left(\mathbb{I}, Y_{x}\right)$, where $Y_{x_{\beta}}=S_{\beta}$ and $Y_{x}$ is a singleton for $x \notin\left\{x_{\beta}: \beta<\alpha\right\}$.

$\operatorname{Dg}_{0} S_{\alpha} \leq 1$ by Theorem 6.5. Proposition 3.2 (2) implies that trind $S_{\alpha} \geq \alpha$, and $S_{\alpha}$ is a chainable continuum with an end point by Proposition 4.2. That $\operatorname{trInd}_{0} S_{\alpha} \leq \alpha$ is shown as in the proof of Theorem 5.7.

7.3. Corollary. There exists a chainable, hereditarily decomposable continuum $S_{\omega\left(\mathfrak{c}^{+}\right)}$with an end point and such that $\operatorname{Dg} S_{\omega\left(\mathfrak{c}^{+}\right)}=\operatorname{Dg}_{0} S_{\omega\left(\mathfrak{c}^{+}\right)}=1$ and trind $S_{\omega\left(\mathfrak{c}^{+}\right)}=\operatorname{trInd}_{0} S_{\omega\left(\mathfrak{c}^{+}\right)}=\omega\left(\mathfrak{c}^{+}\right)$.

Proof. $S_{\omega\left(\mathfrak{c}^{+}\right)}$is constructed as in the proof of Corollary 5.11. We obtain $1 \leq \operatorname{Dg} S_{\omega\left(\mathfrak{c}^{+}\right)} \leq \operatorname{Dg}_{0} S_{\omega\left(\mathfrak{c}^{+}\right)}=1$ by Theorem 6.6.

7.4. Corollary. Let $n \geq 1$ be a natural number, and $\alpha, \beta$ ordinals with $n \leq \alpha \leq \beta \leq \omega\left(\mathfrak{c}^{+}\right)$. Then there exists a continuum $S_{n, \alpha, \beta}$ such that

(a) $\operatorname{dim} S_{n, \alpha, \beta}=n$;

(b) $\operatorname{trDg} S_{n, \alpha, \beta}=\operatorname{trDg} S_{0, \alpha, \beta}=\alpha$;

(c) $\operatorname{trind} S_{n, \alpha, \beta}=\operatorname{trInd}_{0} S_{n, \alpha, \beta}=\beta$;

(d) if $\beta<\omega\left(\mathfrak{c}^{+}\right)$, then $S_{n, \alpha, \beta}$ is separable and first countable;

(e) if $n=1$, then $S_{n, \alpha, \beta}$ is chainable (respectively, hereditarily decomposable).

Proof. We shall construct $S_{1, \alpha, \beta}$ only in the chainable case (an analogous proof using Theorem 5.10 yields HD examples). By Theorem 5.7 or - in case $\alpha=\omega\left(\mathfrak{c}^{+}\right)$- by Corollary 5.11, there exists a chainable continuum $P_{\alpha}$ with an end point $p_{\alpha}$ and such that $\operatorname{trDg} P_{\alpha}=\operatorname{trind} P_{\alpha}=\operatorname{trInd}_{0} P_{\alpha}=\alpha$. By Theorem 7.2 or Corollary 7.3, there exists a chainable continuum $Q_{\beta}$ with an end point $q_{\beta}, \operatorname{Dg} Q_{\beta}=\operatorname{Dg}_{0} Q_{\beta}=1$, and trind $Q_{\beta}=\operatorname{trInd}_{0} Q_{\beta}=\beta$. When we identify the points $p_{\alpha}$ and $q_{\beta}$ in the (disjoint) sum $P_{\alpha} \oplus Q_{\beta}$, we obtain the desired continuum $S_{1, \alpha, \beta}$.

For $n>1$, we take points $p \in \mathbb{I}^{n}$ and $q \in S_{1, \alpha, \beta}$. Identifying $p$ and $q$ in the sum $\mathbb{I}^{n} \oplus S_{1, \alpha, \beta}$, we obtain $S_{n, \alpha, \beta}$.

The rest of this paper is devoted to estimating the Dimensionsgrad of resolutions $S(X)$ where $X$ is made of Euclidean cubes. $\mathbb{H}$ will denote the subspace of $[0,1]^{\mathbb{N}}$ consisting of all points with finitely many non-zero coordinates; it will be equipped with the metric induced by the sup-norm, $\|x\|=\sup _{i \in \mathbb{N}}\left|x_{i}\right|$. For $n=0,1,2, \ldots$, an $n$-cube $Q$ will mean a subspace of $\mathbb{H}$ of the form $Q=\left\{x: a_{i} \leq x_{i} \leq b_{i}\right\}$, where $a_{i} \leq b_{i}$ and the set $J=\left\{i: a_{i}<b_{i}\right\}$ has cardinality $n$. The empty set is regarded as a (-1)-cube. If $Q$ is empty or a singleton, then $\partial Q=\emptyset$, otherwise, $\partial Q$ denotes the combinatorial boundary of $Q$, i.e. the union of the $2 n(n-1)$-cubes $\left\{x \in Q: x_{j}=a_{j}\right\}$ and 
$\left\{x \in Q: x_{j}=b_{j}\right\}, j \in J$. A cube will mean an $n$-cube for some $n$. The intersection of an $n$-cube with an $m$-cube is a cube of dimension $\leq \min \{m, n\}$. A space of type at most $n$ will mean a (necessarily locally compact) subspace of $\mathbb{H}$ that has a locally finite cover consisting of (necessarily countably many) cubes of dimension at most $n$. For a space $Y$ of type at most $n$, we assume that a particular locally finite cover $\mathcal{Q}$ consisting of cubes of dimension at most $n$ has been assigned to $Y$, and we define $\partial Y=\bigcup_{Q \in \mathcal{Q}} \partial Q$. Clearly, $\partial Y$ is a space of type at most $n-1$. A space of type 1 can be readily expressed as a locally finite countable union of arcs any two of which intersect in a finite set, together with a discrete countable subset. The intersection of a space of type at most $m$ with a space of type at most $n$ is a space of type at most $\min \{m, n\}$. In a space $Y$ of type at most $n$, closed balls are of type at most $n$, and every point is the center of arbitrarily small closed balls which are finite unions of cubes of dimension at most $n$. Finally, note that the union of a locally finite collection of spaces of type at most $n$ is a space of type at most $n$.

7.5. LEMmA. If $Y$ is a space of type at most $n \geq 0$, then the collection of all closed subspaces of $Y$ that are of type at most $n-1$ constitutes a strong partition system for $Y$.

Proof. Consider disjoint closed sets $A, B$ of $Y$. Let $L$ be a partition between $A, B$. The locally compact, paracompact Lindelöf space $Y$ has a locally finite open cover $\left\{V_{m}: m \in \mathbb{N}\right\}$, where each $\mathrm{cl} V_{m}$ is compact with either $L \cap \operatorname{cl} V_{m}=\emptyset$ or $(A \cup B) \cap \operatorname{cl} V_{m}=\emptyset$. Take a closed cover $\left\{F_{m}: m \in \mathbb{N}\right\}$ of $Y$ with $F_{m} \subset V_{m}$. As each $F_{m}$ is compact, there is a finite union $B_{m} \subset Y$ of cubes of dimension at most $n$ such that $F_{m} \subset$ int $B_{m} \subset B_{m} \subset V_{m}$. Then $Z=\bigcup\left\{B_{m}: F_{m} \cap L \neq \emptyset\right\}$ is of type at most $n$, and $\partial Z$ is of type at most $n-1$. It is easily seen that $\partial Z$ is a partition in $Y$ between $A$ and $B$.

7.6. Theorem. Let $f: X \rightarrow Y$ be an atomic, fully closed map onto a subspace $Y$ of $\mathbb{H}$ of type at most $n$, where $n$ and $\operatorname{trInd}_{0} f$ are positive and every point-inverse of $f$ contains a point of countable character. Then $\operatorname{trDg}_{0} X \leq \operatorname{trInd}_{0} f+n-1$.

Proof (by induction on $n$ ). For $n=1$, the assertion follows from Theorem 6.5. Let $n>1$. Consider disjoint closed sets $A, B \subset X$. Then $S=f(A) \cap f(B)$ is a discrete zero subset of the perfectly normal space $Y$, and $T=f^{-1}(S)$ is a zero subset of $X$ with $\operatorname{trInd}_{0} T \leq \operatorname{trInd}_{0} f$. Let $E$ be a zero cut in $T$ between $A \cap T$ and $B \cap T$ with $\operatorname{trInd}_{0} E<\operatorname{trInd}_{0} f$. By Lemmas 7.5 and 6.3 , there is a discrete (in $Y \backslash S$ ) collection $\left\{F_{m}: m \in \mathbb{N}\right\}$ consisting of subspaces of $Y \backslash S$ that are closed subspaces of $Y$ of type at most $n-1$ such that $F=E \cup \bigcup_{m \in \mathbb{N}} f^{-1}\left(F_{m}\right)$ constitutes a zero cut in $X$ between $A$ and $B$. As $\left\{F_{m}: m \in \mathbb{N}\right\}$ is a discrete cover of $Z=\bigcup_{m \in \mathbb{N}} F_{m}, Z$ is also of type at most 
$n-1$. By an obvious induction hypothesis, $\operatorname{trDg}_{0} f^{-1}(Z) \leq \operatorname{trInd}_{0} f+n-2$. Note that $\operatorname{trInd}_{0} E \leq \operatorname{trInd}_{0} f+n-2$, and Lemma 2.15 applies, yielding $\operatorname{trDg}_{0} F \leq \operatorname{trInd}_{0} f+n-2$. Hence, $\operatorname{trDg}_{0} X \leq \operatorname{trInd}_{0} f+n-1$ as desired.

7.7. Corollary. For any natural numbers $m$ and $n$, let $Y$ be an $n$ dimensional subspace of $\mathbb{H}$ of type at most $n$, and $f: S \rightarrow Y$ an atomic, fully closed map with $m$-dimensional metric point-inverses. Then

$$
\operatorname{Dg} S=\operatorname{Dg}_{0} S=m+n-1 \text {. }
$$

Proof. By Proposition 5.5, Dg $S \geq m+n-1$ since clearly $\mu(Y) \geq m$. On the other hand, $\operatorname{Ind}_{0} f=m$ and therefore $\operatorname{Dg}_{0} S \leq m+n-1$ by Theorem [7.6.

Note that the corollary holds if $S$ is any weak resolution $S_{X}(Y)$ or any ring-like $R_{X}(Y)$, where $X=\mathbb{I}^{m}, S^{m}$, or $T^{m}$ and $Y=\mathbb{I}^{n}, S^{n}$, or $T^{n}$. On the other hand, in [6] we have proved that for the same $X$ and $Y$, every $S_{X}(Y)$ in the stronger sense has ind $=\operatorname{Ind}_{0}=m+n$.

\section{References}

[1] R. H. Bing, Concerning hereditarily indecomposable continua, Pacific J. Math. 1 (1951), 43-51.

[2] —, Snake-like continua, Duke Math. J. 18 (1951), 653-663.

[3] L. E. J. Brouwer, Über den natürlichen Dimensionsbegriff, J. Reine Angew. Math. 142 (1913), 146-152.

[4] M. G. Charalambous, Two new inductive dimension functions functions for topological spaces, Ann. Univ. Sci. Budapest. Eötvös Sect. Math. 18 (1975), 15-25 (1976).

[5] - A note on the Brouwer dimension of chainable spaces, Topology Appl. 153 (2006), 1271-1278.

[6] M. G. Charalambous and J. Krzempek, Rigid continua and transfinite inductive dimension, ibid. 157 (2010), 1690-1702.

[7] V. A. Chatyrko, On hereditary indecomposable nonmetrizable continua, Mat. Zametki 46 (1989), no. 3, 122-125 (in Russian).

[8] -, On bicompacta with noncoinciding dimensions, Trudy Moskov. Mat. Obshch. 53 (1990), 192-228, 261 (in Russian); English transl.: Trans. Moscow Math. Soc. 1991, 199-236.

[9] —, Classical dimension theory, in: Open Problems in Topology II, E. Pearl (ed.), Elsevier, Amsterdam, 2007, 625-636.

[10] - On properties of subsets of $\left[0, \omega_{\mathfrak{c}}\right] \times I$, Quest. Answers Gen. Topology 26 (2008), 97-104.

[11] V. A. Chatyrko and V. V. Fedorchuk, On the Brouwer dimension of one-dimensional compact Hausdorff spaces, Vestnik Moskov. Univ. Ser. I Mat. Mekh. 2005, no. 2, 22-27, 71 (in Russian); English transl.: Moscow Univ. Math. Bull. 60 (2005), no. 2, $22-27$.

[12] J. F. Davis and W. T. Ingram, An atriodic tree-like continuum with positive span which admits a monotone mapping to a chainable continuum, Fund. Math. 131 (1988), 13-24.

[13] R. Engelking, General Topology, Heldermann, Berlin, 1989. 
[14] R. Engelking, Theory of Dimensions, Finite and Infinite, Heldermann, Lemgo, 1995.

[15] V. V. Fedorchuk, On bicompacta with noncoinciding dimensions, Dokl. Akad. Nauk SSSR 182 (1968), 275-277 (in Russian); English transl.: Soviet Math. Dokl. 9 (1968), 1148-1150.

[16] - On the Brouwer dimension of compact Hausdorff spaces, Mat. Zametki 73 (2003), 295-304 (in Russian); English transl.: Math. Notes 73 (2003), 271-279.

[17] —, An example of a compact Hausdorff space with different Lebesgue, Brouwer, and inductive dimensions, Mat. Sb. 195 (2004), no. 12, 109-122 (in Russian); English transl.: Sb. Math. 195 (2004), 1809-1822.

[18] —, Fully closed maps and their applications, J. Math. Sci. (N.Y.) 136 (2006), 42014292; Russian original: Fundam. Prikl. Mat. 9 (2003), no. 4, 105-235.

[19] V. V. Fedorchuk, M. Levin, and E. V. Shchepin, On the Brouwer definition of dimension, Uspekhi Mat. Nauk 54 (1999), no. 2, 193-194 (in Russian); English transl.: Russian Math. Surveys 54 (1999), 432-433.

[20] V. V. Fedorchuk and J. van Mill, Dimensionsgrad for locally connected Polish spaces, Fund. Math. 163 (2000), 77-82.

[21] A. V. Ivanov, The dimension of not perfectly normal spaces, Vestnik Moskov. Univ. Ser. I Mat. Mekh. 31 (1976), no. 4, 21-27 (in Russian); English transl.: Moscow Univ. Math. Bull. 31 (1976), no. 3-4, 64-69.

[22] J. Krzempek, Fully closed maps and non-metrizable higher-dimensional AndersonChoquet continua, Colloq. Math. 120 (2010), 201-222.

[23] -, Components and inductive dimensions of compact spaces, Czechoslovak Math. J. 60 (2010), 445-456.

[24] W. Lewis, The pseudo-arc of pseudo-arcs is unique, Houston J. Math. 10 (1984), 227-234.

[25] S. Mazurkiewicz, Sur les courbes d'ordre c, Fund. Math. 16 (1930), 337-347.

[26] J. van Mill, The Infinite-Dimensional Topology of Function Spaces, Elsevier, Amsterdam, 2002.

[27] B. A. Pasynkov, On dimension theory, in: Aspects of Topology, I. M. James and E. H. Kronheimer (eds.), London Math. Soc. Lecture Note Ser. 93, Cambridge Univ. Press, Cambridge, 1985, 227-250.

Michael G. Charalambous

Department of Mathematics

University of the Aegean

83 200, Karlovassi, Samos, Greece

E-mail: mcha@aegean.gr
Jerzy Krzempek

Institute of Mathematics Silesian University of Technology

Kaszubska 23

44-100 Gliwice, Poland E-mail: j.krzempek@polsl.pl

Received 12 June 2009;

in revised form 4 June 2010 